\title{
Algoritmos genéticos para detecção de dano estrutural
}

\author{
Herbert Martins Gomes ${ }^{(1)}$, Newton R. Santos da Silva ${ }^{(2)}$
}

Trabalho recebido em 07/11/2005 e aprovado para publicação em 20/03/2006.

\section{Resumen}

A última década presenciou o desenvolvimento de um grande número de testes não destrutivos para a avaliação da integridade estrutural. Este crescimento é devido ao crescente interesse em reduzir o tempo e os custos no monitoramento e na manutenção preditiva. Desta forma, diversos métodos concebidos para detectar o dano estrutural foram propostos baseados, por exemplo, em análise de sensibilidade ou métodos estatísticos. Entretanto, alguns destes métodos apresentam problemas práticos na medição das características dinâmicas estruturais tais como as formas modais. Alguns outros métodos baseados exclusivamente nas respostas estruturais mostram desvantagens para encontrar a localização do dano na estrutura. Métodos baseados na resposta estrutural assim como em modelos paramétricos calibrados têm demonstrado serem mais robustos nestas tarefas. Seguindo esta direção, este trabalho trata de uma metodologia baseada em Algoritmos Genéticos para detectar falha através de medições experimentais das freqüências naturais com o auxílio de um modelo numérico paramétrico. Alguns exemplos são apresentados na detecção de danos em múltiplos locais e intensidades. São enfatizadas as vantagens desta metodologia devido à pouca quantidade de informação necessária assim como a sua robustez.

Palavras-chave: algoritmos genéticos, detecção de dano, identificação de danos

1 Prof. Dr., PROMEC/UFRGS. Programa de Pós Graduação em Engenharia Mecânica, Universidade Federal do Rio Grande do Sul, Rua Sarmento Leite, 425, sala 314, 3o andar, 90050-170, Porto Alegre, RS, Brasil. Fone: +5551 3316-3255. Fax: +55 513316 - 4001. E-mail: herbert@mecanica.ufrgs.br

2 Aluno PROMEC/UFRGS. Programa de Pós Graduação em Engenharia Mecânica, Universidade Federal do Rio Grande do Sul, Rua Sarmento Leite, 425, sala 314, 3o andar, 90050-170, Porto Alegre, RS, Brasil. Fone: +55 51 3316-3255. Fax: +55 513316 - 4001. E-mail: newtonrss@yahoo.com.br 


\section{Introdução e objetivo}

A detecção de dano em estruturas tem sido pesquisada por diversos autores. Em particular, as técnicas de detecção baseadas em ensaios não destrutivos (Non Destructive Testing, NDT) têm sido preferidas devido ao baixo custo e a aspectos operacionais relacionados ao uso da estrutura analisada durante os ensaios. Há métodos para detecção de dano baseados em análise de sensibilidade e parâmetros estatísticos de alguma resposta da estrutura frente à excitações. Alguns outros métodos são baseados nas características dinâmicas da estrutura tais como freqüências naturais, formas modais e amortecimento estrutural. Estes métodos têm tomado vantagem do desenvolvimento atual das técnicas de análise modal com medições acuradas destes parâmetros modais. Quando o evento de dano ocorre, as características dinâmicas estruturais são modificadas e podem ser usadas como indicadores de danos.

Como indicado por Friswell e Penny (1997), Redes Neurais Artificiais e Algoritmos Genéticos têm sido vistos como potenciais ferramentas para o tratamento de problemas difíceis na localização do dano. Como colocado pelos autores, o método não trata a raiz do problema. A grande vantagem em usar, por exemplo, algoritmo genético, está na habilidade de encontrar mínimos globais em otimizações difíceis onde há muitos mínimos locais como no caso da localização do dano. Para algumas aplicações em redes neurais em detecção de dano, o treinamento dos cenários de dano deve ser avaliado previamente, o qual requer a existência de modelos paramétricos bem calibrados com a estrutura analisada. Para ambos os métodos uma razoável quantidade de cálculos é necessária.

Neste trabalho, um algoritmo genético com codificação real é utilizado conjuntamente com um modelo de elementos finitos de barras para determinar tanto a localização quanto a intensidade do valor do dano em estruturas. Para isto, as mudanças nas freqüências naturais da estrutura na presença do dano em relação às suas freqüências naturais sem dano são empregadas como parâmetro a ser otimizado pelo algoritmo genético na procura da estrutura de elementos finitos que melhor representa estas mudanças.

\section{Detecção de dano como um problema de identificação de parâmetros}

Raie e Rashtchi (2002) propuseram um método de deteç̧ão de dano para as voltas do enrolamento de um motor de indução baseado na identificação de parâmetros usando um algoritmo genético. Foi usado um algoritmo genético com codificação em número reais e um modelo numérico para as tensões, correntes no estator do motor de indução e sua velocidade. O novo método de detecção da magnitude e determinação de voltas curtas no enrolamento do estator dos motores de indução foi validado pela geração de falhas nas voltas do enrolamento e minimizando o quadrado das diferenças entre os resultados experimentais e os obtidos pelo algoritmo genético. A identificação e avaliação da magnitude da falha foram obtidas com sucesso. 
Chou e Ghaboussi (2001) propuseram duas opções de uso de algoritmos genéticos para a avaliação do dano baseada na medição de deslocamentos. O primeiro usa a medida dos deslocamentos da estrutura, a qual deve ser minimizada com os correspondentes deslocamentos medidos por elementos finitos. O segundo evita a solução custosa do sistema de equações numa análise por elementos finitos. Isto é feito codificando os deslocamentos não medidos com as propriedades dos materiais numa cadeia de cromossomos e deixando os valores dos deslocamentos não medidos evoluírem junto com os deslocamentos medidos. Isto resulta na combinação das matrizes de rigidez e vetores de deslocamentos para produzir as mesmas respostas estáticas. Para implementar isto, foi proposto um esquema de escalonamento para normalizar os deslocamentos não medidos com os deslocamentos medidos. Através de uma série de exemplos simples com uma treliça plana o método proposto foi capaz de detectar e localizar com êxito a posição e a magnitude das falhas e também corretamente determinar o valor dos deslocamentos nodais não medidos, evitando uma completa análise por elementos finitos.

Sazonov, Klinkhachorn e Hatabe (2002) usaram algoritmos genéticos para produzir um filtro de amplitudes suficientemente otimizado para extrair informações da energia de deformação das formas modais. Um modelo de elementos finitos foi usado para produzir um conjunto de dados de treinamento com as localizações do dano conhecidas. A amplitude característica do filtro foi codificada como um cromossomo de um algoritmo genético onde o coeficiente de passagem para cada harmônico da sua Transformada Discreta de Fourier (TDF) era um número entre 0 e 1 com uma resolução de 8 bits (Gray code). A otimização genética foi feita baseada na minimização da razão entre sinal e a distorção. De acordo com os autores os resultados obtidos pelo AG confirmou as predições teóricas e permitiu o melhoramento nos métodos baseados em sensibilidade para danos de pequena magnitude.

Ostachowicz, Krawczuk e Cartmell (1996) fizeram uma série de testes com algoritmos genéticos, usado como uma ferramenta de maximização, para detectar a localização da delaminação numa viga compósita engastada. O índice DLAC foi usado como função objetivo e um gene de 8 bits e cromossomo de 33 posições, sendo 11 deles para cada variável a ser otimizada, utilizado para a localização do dano em dois locais e a profundidade da camada danificada. De acordo com os autores, os resultados são promissores, particularmente devido ao fato de que o número de cálculos necessários para a detecção da falha é muito menor que aqueles requeridos por algoritmos de procura clássicos.

Friswell, Penny e Garvey (1998) aplicaram algoritmos genéticos ao problema de detecção de dano usando dados de vibração. O método foi utilizado para otimizar as variáveis de localização discretas. Um método padrão de sensibilidade dos autovalores foi usado para otimizar a intensidade do dano. Dois exemplos foram usados para validar o método proposto: uma barra engastada simulada numericamente e uma placa engastada analisada experimentalmente. A função objetivo adotada foi uma mistura de termos relacionados às diferenças nas freqüências naturais, um termo relacionado a erros nas formas modais e um termo para penalizar presença de dois locais de dano. 
O último termo foi introduzido devido à tendência de freqüentemente o algoritmo encontrar danos em quase todos os locais devido aos ruídos presentes nas medidas e às incertezas do modelo paramétrico.

Ratman e Rao (2004) implementaram um programa computacional para o método da rigidez em conjunto com um algoritmo genético para detectar dano em vigas e estruturas treliçadas. No seu trabalho, o método das forças foi utilizado como uma função objetivo a ser minimizada por algoritmos genéticos. Este método usou a equação associada de autovalores e autovetores para definir um resíduo de forças, o qual deveria ser minimizado. Trabalho semelhante foi desenvolvido por Genovese et al. (2002) usando redes neurais artificiais ao invés de algoritmos genéticos. Em ambos os casos, como esperado, um modelo paramétrico bem calibrado da estrutura (que forneça as mesmas respostas que a estrutura real) deve ser construído.

Tebaldi et al. (2004) propôs uma metodologia de identificação de falhas trabalhando no domínio da freqüência primeiramente usando o método da matriz de impedância dinâmica para detectar falha em locais e num segundo estágio a intensidade da falha foi tratada por algoritmos genéticos. Basicamente as Funções de Resposta em Freqüência (FRF) experimentais foram ajustadas numericamente àquelas numéricas obtidas por um modelo em elementos finitos. A função objetivo a ser minimizada foi a diferença entre as FRF, através da otimização. $O$ exemplo usado foi o de uma viga simples avaliada numericamente em elementos finitos e as falhas foram simuladas numericamente em dois locais e implementadas simultaneamente. Como comentários finais, eles concluíram que a aplicação combinada da matriz de impedância junto com técnicas de AG pode oferecer um critério robusto e eficiente para a identificação de dano estrutural, uma vez que o conjunto de parâmetro para o processo de otimização foi drasticamente reduzido.

\section{Fundamentos de algoritmos genéticos}

Algoritmos Genéticos (GA) são técnicas de otimização baseadas na "Teoria da Evolução" e na sobrevivência do ser mais apto. Estes algoritmos pertencem a uma classe mais abrangente de algoritmos de otimização chamados de algoritmos de procura estocástica. A teoria de Darwin da Seleção Natural diz que: “... qualquer ser, se ele modifica-se ligeiramente de maneira proveitosa a si mesmo, terá melhores chances de sobrevivência...". Os GA simulam o processo evolutivo numericamente. Eles representam os parâmetros de um dado problema codificando-os num vetor. Como na genética, genes são constituídos por cromossomos. Similarmente, em GA na sua forma simplificada, os vetores dos parâmetros (variáveis a serem otimizadas no processo de otimização) são codificados em "bits" (seqüências de uns e zeros). Um vetor de "bits" pode ser decodificado no respectivo valor do parâmetro do problema e a total avaliação do vetor de "bits" para um indivíduo (candidato à resposta do problema de otimização) pode ser ponderada seguindo-se alguma função de aptidão representativa das características (fenótipo) do indivíduo para aquele conjunto de "bits". 
Um algoritmo genético simples constitui-se de três operadores básicos, sendo eles a reprodução, o "crossover" ou recombinação e a mutação. O algoritmo começa com uma população de indivíduos, cada um deles representando uma possível solução para 0 problema. Os indivíduos, como na natureza, se utilizam destes três operadores básicos e evoluem em gerações onde a teoria de Darwin para a esta evolução prevalece. Em outras palavras, uma população de indivíduos mais adaptados surge como uma seleção natural do processo ao longo das gerações. Ao nível da reprodução, a avaliação da função objetivo (aptidão) indica quais indivíduos irão ter mais chances de procriar e gerar uma prole maior nas gerações seguintes.

Nas operações genéticas, os genes de pares de indivíduos são trocados e como na natureza, esta troca pode se dar de diversas formas sendo então comumente chamadas de "crossover" ou recombinação.

As diferenças básicas entre as técnicas convencionais de otimização e os algoritmos genéticos (GA) podem ser resumidas como segue (Goldberg, 1989):

- Os GA operam de forma codificada na tarefa de procura dos parâmetros;

- Os GA trabalham com uma população codificada a qual representa os valores numéricos de uma variável em particular;

- Diferentemente da maioria dos algoritmos de otimização, os quais requerem avaliações das derivadas da função objetivo, os GA somente requerem o uso do valor da função objetivo;

- Somente regras probabilísticas e a regra da seleção natural são utilizadas com os GA.

A representação binária tem uma importância histórica devido ao primeiro uso por Holland (1975). Quando se trabalha com o algoritmo genético na forma binária, cada um dos parâmetros reais a serem otimizados são traduzidos num código binário seguindo a seguinte equação:

$$
s=\operatorname{bin}_{n}\left\{\operatorname{round}\left(2^{n}-1\right) \frac{\left[b_{i}(k)-P_{\min }(k)\right]}{\left[P_{\max }(k)-P_{\min }(k)\right]}\right\}
$$

onde $\operatorname{bin}_{n}$ indica a tradução binária para um vetor binário $s$ de $n$ "bits", $n$ significando o número de "bits", $\mathrm{P}(\mathrm{k})$ significando os limites máximos e os mínimos permitidos para cada variável e $k$ o indivíduo em específico.

Para transformar o código binário para valores reais a seguinte equação é utilizada como indicado em seqüência:

$$
b_{i}(k)=P(k)_{\min }+\operatorname{bin}^{-1}(s) \frac{P(k)_{\max }-P(k)_{\min }}{2^{n}-1}
$$

onde $\operatorname{bin}^{-1}(s)$ significa a tradução dos valores codificados em binário para os respectivos valores reais. Deve ser notado que com esta formulação está implícito que o mapeamento tem uma resolução de $\left[P(k)_{\max }-P(k)_{\min }\right] /\left(2^{n}-1\right)$. Isto restringe a procura no espaço dos pa- 
râmetros reais a valores discretos os quais podem induzir a obtenção de ótimos locais.

Isto pode ser contornado utilizando-se um algoritmo genético com codificação real. Esta aproximação assume valores reais para cada variável. As principais mudanças para o algoritmo com codificação binária são encontradas no operador de "crossover" (recombinação). Há diversas formas de tratar com a recombinação em algoritmos genéticos com codificação real tais como: a recombinação plana, a recombinação simples, a recombinação aritmética, a recombinação de Wright, a recombinação BGA, etc. Neste trabalho a recombinação BLX- $\alpha$ ("blended crossover") é utilizada devido ao fato dela operar inicialmente com uma fase exploratória abrangente do campo de parâmetros seguida de uma fase de pesquisa mais detalhada para a melhoria da resolução dos parâmetros. Ela pode ser descrita como:

$$
\begin{aligned}
& \Delta=\max \left[b_{i}(k), b_{i+1}(k)\right]-\min \left[b_{i}(k), b_{i+1}(k)\right] \\
& b(k)=\operatorname{random}\left\{\min \left[b_{i}(k), b_{i+1}(k)\right]-\alpha \Delta, \max \left[b_{i}(k), b_{i+1}(k)\right]+\alpha \Delta\right\}
\end{aligned}
$$

onde, i e i+1 estão referidos a dois cromossomos de indivíduos, $\alpha$ significa um parâmetro de diminuição da exploração e random significa um número aleatório no respectivo intervalo. A Tabela 1 resume os principais passos seguidos por um algoritmo genético com codificação real para maximizar funções.

\subsection{Detecção de Dano como um problema de Otimização}

Messina et al(1998) propuseram um critério para a identificação de múltiplos danos locais (MDLAC - Multiple Damage Location Assurance Criteria) o qual avalia a correlação entre os valores numéricos de um modelo paramétrico e os resultados experimentais das variações de freqüências devido ao dano. 
Tabela 1: Algoritmo genético com codificação real utilizado.

Inicialize o tempo $\mathrm{t}=0$

Inicialize o tamanho da população "m", Probabilidade de Mutação "Pm", Probabilidade de recombinação "Pc", Número de cromossomos individuais "nc", Limites permitidos para cada cromossomo, "Pmáx(nc), Pmín(nc)".

Geração da população inicial $B_{0}=\left(b_{1,0}, b_{2,0}, \ldots, b_{m, 0}\right)$

"Laço sobre as Gerações"

Enquanto a condição de parada não for satisfeita

"Seleção Proporcional"

Laço $\mathrm{i}=1$ até $\mathrm{m}$

$\mathrm{x}=\operatorname{random}(0,1)$

$\mathrm{k}=1$

Enquanto $\mathrm{k}<\mathrm{m}$ e $\mathrm{x}<\sum_{j=1}^{k} f\left(b_{j, t}\right) / \sum_{j=1}^{m} f\left(b_{j, t}\right)$

$\mathrm{k}=\mathrm{k}+1$

$b_{i, t+1}=b_{k, t}$

Fim Enquanto

Fim do laço

"Recombinação em um ponto"

Laço $\mathrm{i}=1$ até $\mathrm{m}-1$ passo 2

Se random $(0,1)<$ Pc então

$$
\begin{aligned}
& \alpha=0.25 \\
& \Delta=\max \left[b_{i, t}(k), b_{i+1, t}(k)\right]-\min \left[b_{i, t}(k), b_{i+1, t}(k)\right] \\
& b_{i, t+1}(k)=\operatorname{random}\left\{\min \left[b_{i, t}(k), b_{i+1, t}(k)\right]-\alpha \Delta, \max \left[b_{i, t}(k), b_{i+1, t}(k)\right]+\alpha \Delta\right\} \\
& b_{i+1, t+1}(k)=\operatorname{random}\left\{\min \left[b_{i, t}(k), b_{i+1, t}(k)\right]-\alpha \Delta, \max \left[b_{i, t}(k), b_{i+1, t}(k)\right]+\alpha \Delta\right\}
\end{aligned}
$$

Fim Se

Fim do Laço

"Mutação dos filhos"

Laço $\mathrm{i}=1$ até $\mathrm{m}$

Se random $(0,1)<$ Pm então

$\mathrm{k}=\operatorname{random}(0,1) * \mathrm{nc}$

$b_{i, t+1}(k)=\operatorname{random}\left\{P_{\max }(k), P_{\min }(k)\right\}$

Fim Se

Fim do Laço

Fim do Enquanto

$$
\operatorname{MDLAC}(\delta \mathbf{D})=\frac{\left|\Delta \boldsymbol{\Omega}^{T} . \delta \boldsymbol{\Omega}(\delta \mathbf{D})\right|^{2}}{\left(\Delta \boldsymbol{\Omega}^{T} . \Delta \boldsymbol{\Omega}\right) \cdot\left[\delta \boldsymbol{\Omega}(\delta \mathbf{D})^{T} \delta \boldsymbol{\Omega}(\delta \mathbf{D})\right]}
$$

onde $\delta \mathbf{D}$ representa o vetor de dados em cada elemento, $\Delta \mathbf{\Omega}$ as variações de freqüências obtidas experimentalmente e $\delta \boldsymbol{\Omega}$ as variações de freqüências do modelo paramétrico e eu são função do vetor de danos. O índice MDLAC assume valor 1.0 quando houver completa correlação entre as variações de freqüência dos resultados numéricos e os resultados experimentais e 0.0 para dados não correlacionados. Os mesmos autores propõem uma aproximação de $2^{\underline{a}}$ ordem para levar em conta relações não lineares 
entre mudanças de freqüências e a extensão do dano. Esta aproximação de $2^{a}$ ordem envolve a solução de um equação de $2^{\circ}$ grau para avaliar o nível do dano. Os respectivos coeficientes finais de escala para o dano em cada elemento são obtidos por uma média destes valores para cada modo.

A principal diferença entre o critério de múltiplos danos proposto por Messina (1998) e o critério aqui proposto é que a avaliação do vetor de variações não usa aproximações de $1^{\mathrm{a}}$ ou $2^{\mathrm{a}}$ ordem mas uma análise de elementos finitos completa para o correspondente vetor de dano. Isto permite que os efeitos não lineares do dano de grande intensidade nas freqüências naturais sejam filtrados, uma vez que a análise completa de elementos finitos é feita.

Este índice é usado como função objetivo a ser maximizada no processo de otimização que é feito através do algoritmo genético. Então, cada indivíduo da população tem um gene composto por cromossomos que representam a combinação do vetor de solução, que é um vetor de valores reais contendo os índices de dano para cada elemento na estrutura. Associado à cada indivíduo há uma função de aptidão avaliada de acordo com a Eq. (1). Esta população evolui por gerações onde a Teoria da Seleção Natural é empregada.

\section{Exemplo}

Este exemplo mostra a robustez do algoritmo proposto para a detecção tanto em localização quanto em intensidade do dano. Este exemplo inclui uma viga simplesmente apoiada com seção transversal retangular, altura $h=0,24 \mathrm{~m}$, largura $\mathrm{b}=0,14 \mathrm{~m}$ e comprimento de $\mathrm{L}=2,4 \mathrm{~m}$. O Módulo de Elasticidade do material usado foi de $\mathrm{E}=2,5 \times 10^{10} \mathrm{~N} / \mathrm{m}^{2}$ e a densidade do material empregado foi de $\rho=2,5 \times 10^{3} \mathrm{~kg} / \mathrm{m}^{3}$. A característica simplificada e dimensões deste exemplo assemelham-se à de um protótipo em escala reduzida que será testado em laboratório e que brevemente será usado como padrão para validação para os algoritmos aqui propostos.

A Figura 1 mostra um esquema das dimensões estruturas e da numeração para a discretização dos elementos usada na análise por elementos finitos. Foi utilizado um modelo de viga com seis graus de liberdade por nó, restringidos no plano da estrutura, resultando em três graus de liberdade por nó (duas translações e uma rotação).

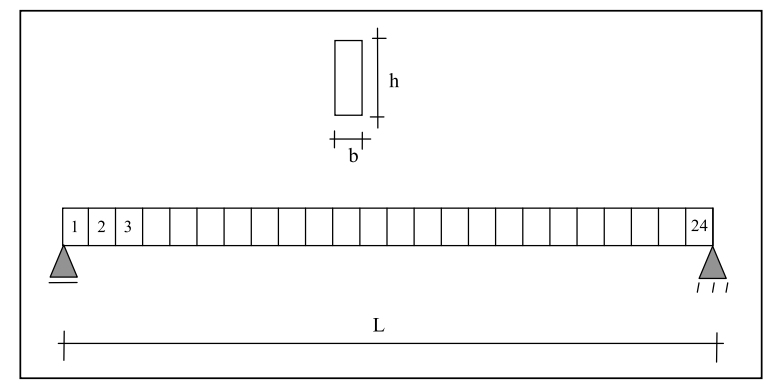

Figura 1: Viga simplesmente apoiada com seção transversal e dimensões 
Como parâmetros usados no algoritmo para este exemplo adotou-se um tamanho de população de $\mathrm{m}=50$ indivíduos, o número de cromossomos por indivíduo (estrutura) $\mathrm{nc}=24$ (dano em cada elemento discretizado da estrutura), uma probabilidade de Crossover de $\mathrm{Pc}=100 \%$ (todos os indivíduos terão chances de gerar prole), uma probabilidade de mutação de $\mathrm{Pm}=1 \%$ (um em cada 100 filhos sofrerá uma mutação), limites para a intensidade do dano entre 0.0 e 0.9 (iguais para todas as 24 variáveis a serem otimizadas), número máximo de gerações de 1000. Por fim, como limite para parada do processo de otimização, adotou-se o valor da Diversidade (Coeficiente de Variação $\sigma / \mu$ das funções de aptidão dos indivíduos de determinada geração) menor que $1 \times 10^{-3}$ como indicativo de convergência.

Para investigar o algoritmo, foi inicialmente assumido um cenário de dano numericamente gerado no elemento 9 com 10\% de dano (a propriedade de inércia Iz foi reduzida desta quantidade). As primeiras cinco freqüências naturais da estrutura danificada foram usadas como entrada para o algoritmo genético para maximizar o índice MDLAC.

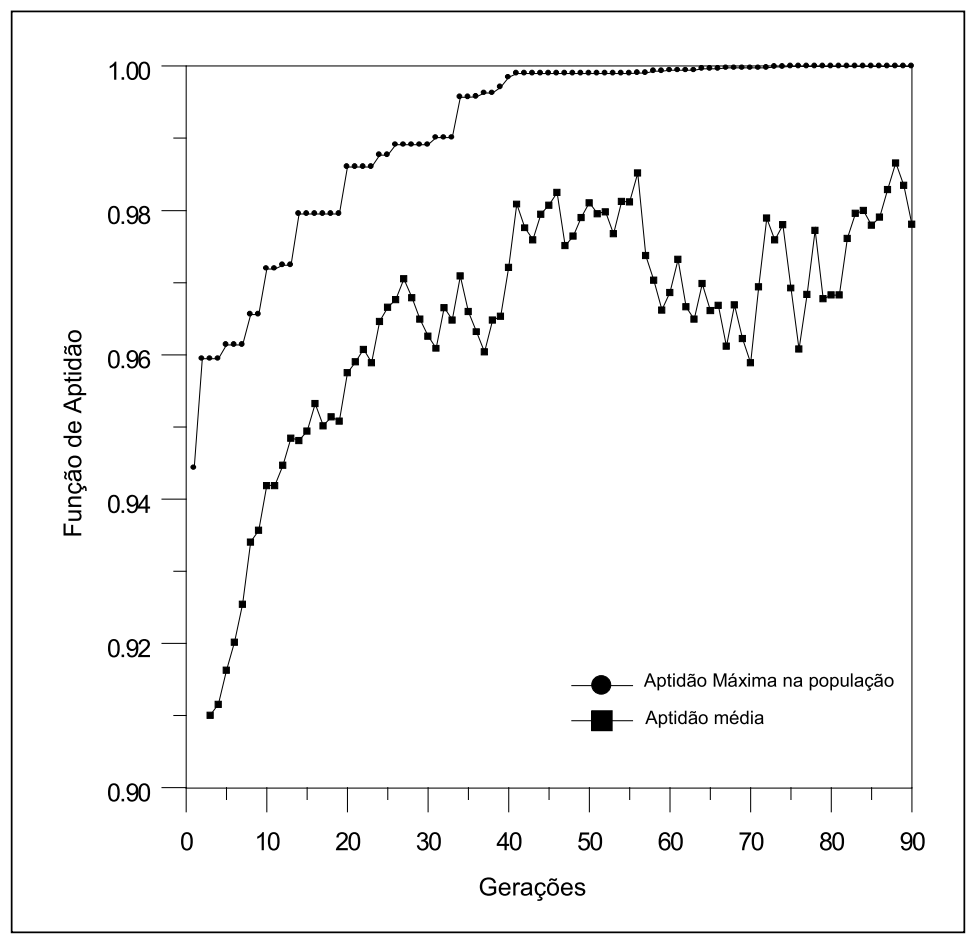

Figura 2: Evolução da média da aptidão (MDLAC) da população e melhor aptidão com as gerações.

A figura 3 mostra a evolução da intensidade do dano nos elementos com as gerações para a média da população e para o melhor indivíduo da população. Pode ser visto que na $37^{\mathrm{a}}$. geração o local do dano é identificado e o valor da intensidade deste dano é corretamente avaliado posteriormente na $90^{\mathrm{a}}$ geração. 


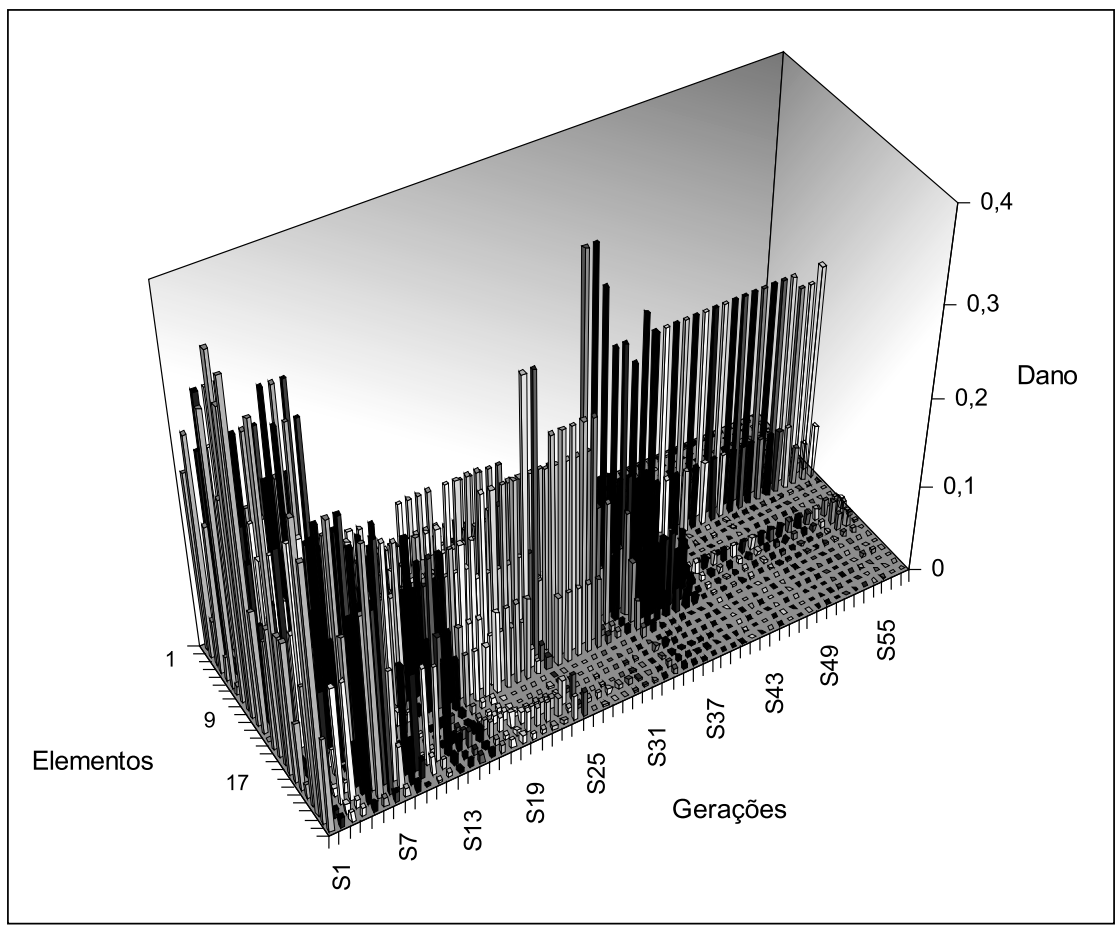

Figura 3: Evolução da intensidade do dano no melhor indivíduo com as gerações.

Como indicado na Tabela 1 , foram investigados mais seis cenários de dano na viga. Foi permitida a ocorrência do dano em diferentes elementos e em mais de um lugar ao mesmo tempo.

Tabela 2: Cenários de dano analisados.

\begin{tabular}{cccccccc}
\hline Cenários & 1 & 2 & 3 & 4 & 5 & 6 & 7 \\
\hline Elemento -Dano(\%) & $3-10 \%$ & $13-10 \%$ & $22-10 \%$ & $16-10 \%$ & $9-5 \%$ & $9-1 \%$ & $2,13,22-10 \%$ \\
\hline
\end{tabular}

Os casos indicados na Tabela 1 foram simulados numericamente e as primeiras cinco freqüências naturais foram usadas para alimentar o Algoritmo Genético com base na função objetivo construída. A Figura 4 mostra os resultados para os cenários testados. Com exceção do caso 7, todos os outros cenários foram corretamente localizados e a intensidade do dano corretamente avaliada. Neste $7^{\circ}$ caso a intensidade do dano foi corretamente avaliada, mas a localização do mesmo resultou num elemento ao lado da posição correta, como indicado pela Figura 4. 


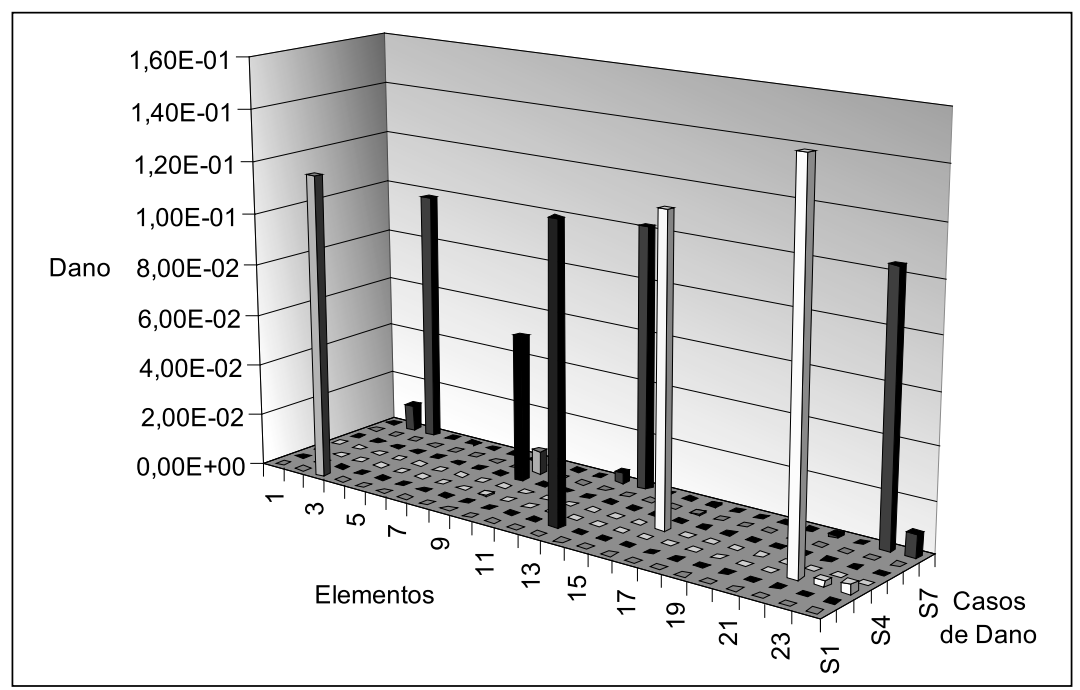

Figura 4: Cenários de dano e previsões numéricas.

\section{Conclusões}

Neste trabalho apresentou-se uma metodologia para avaliar a intensidade do dano assim como a sua localização apenas pelas mudanças nas freqüências naturais. A formulação usa um algoritmo genético com codificação real como uma ferramenta de otimização para ajustar as freqüências naturais de um modelo em elementos finitos paramétrico com as freqüências experimentais através do índice MDLAC.

A metodologia tem mostrado um comportamento robusto, uma vez que poucas aproximações são feitas (discretização em elementos finitos ou hipóteses de linearidade para o modelo numérico) no comportamento estrutural dinâmico. Na forma em que se encontra, o modelo comporta facilmente a introdução de não linearidades que se acharem importantes. Esta abordagem contorna problemas de não linearidade entre as mudanças nas freqüências naturais e o aumento do dano, presente em alguns modelos baseados na sensibilidade modal. Por outro lado, o custo computacional pode ser aumentado juntamente com a complexidade do modelamento (discretização) do comportamento estrutural.

Cenários de danos com intensidade moderada foram encontrados com sucesso usando somente as primeiras cinco freqüências naturais. A intensidade dos danos quando ocorrem em mais de um ponto da estrutura foram corretamente identificados, mas a previsão de seu posicionamento não foi tão preciso quanto o esperado.

Ainda assim, alguns melhoramentos na ferramenta de otimização podem ser implementados e novos modelos estruturais podem ser testados. Alguns testes experimentais estão sendo feitos e serão posteriormente adicionados em trabalhos futuros nas comparações para a validação do modelo. 


\section{Referências}

Au, F.T.K.; Cheng, Y.S.; Tham, L.G. and Bai, Z.Z. (2003). Structural damage detection based on a micro-genetic algorithm using incomplete and noisy modal test data. Journal of Sound and Vibration, v. 259, n. 5, p. 1081-1094.

Chou, J.H. and Gaboussi, J. (2001). Genetic algorithm in structural damage detection. Computers and Structures, v.79, p.1335-1353.

Doebling, S.W.; Farrar, C.R.; Prime, M. B. and Shevitz, M.B.D.W. (1996). Damage identification and health monitoring of structural and mechanical systems from changes in their vibration characteristics: A literature review. Los Alamos National Laboratory Report, LA-13070-MS.

Friswell, M.I. and Penny, J.E.T. (1997). Is damage Location using vibration measurements practical? Proceedings of the EUROMECH 365, International Workshop: DAMAS 97, Structural Damage Assessment Using Advanced Signal Processing Procedures, Sheffield, UK, June/July.

Friswell, M.I.; Penny, J.E.T.; Garveyb, S.D. (1998). A combined genetic and eigensensitivity algorithm for the location of damage in structures. Computers and Structures, v. 69, p. 547-556.

Genovese, M.; Brito, J. L. V. and DOZ, G. N. (2002). Identificação de danos mediante a utilização de um método híbrido entre o método do erro e a técnica de redes neurais artificiais. In: XXX Jornadas Sul Americanas de Engenharia Estrutural, Brasília, Universidade de Brasília, UnB., DF, 11p.

Goldberg, D. E. (1989). Genetic Algorithms in search, optimization and machine learning. AddisonWesley Publishing, Reading, MA.,USA

Holland, J. (1975). Adaptation in natural and artificial systems. University of Michigan Press, Ann Arbor, MI., USA

Messina, A.; Williams, E. J. and Contursi, T. (1998). Structural damage detection by a sensitivity and statistical-based method. Journal of Sound and Vibration, v. 216, n. 5, p.791-808.

Ostachowicz, W. M.; Krawczuk, M. and Cartmell, M. P. (1996). Genetic algorithms in health monitoring of structures. University of Glasgow, Department of Mechanical Engineering, James Watt Building, Glasgow, Scotland, Internal Report.

Pawar, M. and Ganguli, R. (2003). Genetic fuzzy system for damage detection in beams and helicopter rotor blades. Computational Methods in Applied Mechanical Engineering, v. 192, p. 2031-2057.

Raie, A.; Rashtchi, V. (2002). Using a genetic algorithm for detection and magnitude determination of turn faults in an induction motor. Electrical Engineering Department, Amirkabir University of Technology, Tehran, Iran, Internal Report.

Rao, M. A.; Srinivas J. and Murthy, B. S. N. (2004). Damage detection in vibrating bodies using genetic algorithms. Computers and Structures, v. 82, p. 963-968.

Ratman. C. and Rao, D. V. P. (2004). Identification of damage in structures using genetic algorithms. IE (I) Journal-MC, v. 84, p.154-160.

Samanta, B. (2004). Artificial neural networks and genetic algorithms for gear fault detection. Mechanical Systems and Signal Processing, v. 18, p. 1273-1282.

Sazonov, E. S.; Klinkhachorn, P. and Halabe, U. B. (2002). Genetic Algorithms based parameter optimization of a non-Destructive damage detection method. IEEE Transactions.

Silva, M. F. T.; Borges, L. M. S. A.; Rochinha, F. A. and Carvalho, L. A. V. (2002). Genetic algorithms for identification of elastic constants of composite materials. In: 4th. International Conference on Inverse Problems in Engineering, Rio de Janeiro, Brazil.

Tebaldi, A.; Lopes Jr, V. and Aparecido, J. B. (2004). Fault detection using piezoelectric devices and genetic algorithms. In: Inverse Problems, Design and Optimization Symposium, Rio de Janeiro, Brazil. 


\title{
Genetic algorithms for structural damage detection
}

\begin{abstract}
Abstrac
The last decade witnessed the development of a large number of non-destructive tests for structural integrity evaluation. This growth is due to attracted interest to reduce time and costs in performing damage monitoring and predictive maintenance. In this way several methods intended to detect structural damage based on sensitivity and statistical methods were proposed. However, some of these methods present some practical problems in measuring structural dynamic characteristics such as dynamic mode shapes. Some methods based exclusively on structural responses have shown disadvantages in finding the damage position on structures. Methods based on structural responses as well as tuned structural parametric models have been demonstrated more robust in this task.
\end{abstract}

Keywords:

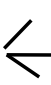

\section{Objective}

This paper deals with a genetic algorithm based methodology to detect faults through experimental measurements of the natural frequencies with assist of a parametric structural model. Some examples are presented to damage detection on multiple sites and levels on a structure. It is emphasized the advantages of this methodology due to the little amount of information necessary as well as its robustness.

\section{Background}

Messina et al(1998) have proposed a multiple damage location assurance criterion (MDLAC) to evaluate the correlation between experimental and numerical values of frequency variations due to damage as indicated by Equation (4).

The MDLAC index assumes 1.0 for fully correlated numerical and experimental frequency changes measured, and 0.0 for non-correlated ones. The main difference between the Multiple Damage Location Assurance Criterion proposed by Messina (1998) and the criteria proposed here is that the evaluation of the $\delta \Omega(\delta \mathbf{D})$ vector does not uses first or second order approximations, but actually a finite element run for the corresponding damage $\delta \mathbf{D}$ vector. This enables that non linear effects of large damage on natural frequencies are filtered out since an actual finite element run is accomplished. 
This index is used as the objective function to be maximized in the optimization process carried out by genetic algorithms. So, each individual of the population has a gene composed by chromosomes that represents a combination of the solution vector, that is, a vector of real values containing the damage indexes for each element in the structure. Associated to each individual there are a fitness function evaluated as indicated by Equation (1). This population evolves by generations where Theory of Natural Selection is employed.

\section{Evaluation metodology}

The MDLAC index is used as the objective function to be maximized in the optimization process carried out by genetic algorithms. So, each individual of the population has a gene composed by chromosomes that represents a combination of the solution vector, that is, a vector of real values containing the damage indexes for each element in the structure. Associated to each individual there are a fitness function evaluated as indicated by Equation (1). This population evolves by generations where Theory of Natural Selection is employed.

\section{Results}

The example of the simple span beam shows the robustness of the proposed algorithm in detecting as much in location as in intensity the damage. The beam has a rectangular cross sectional area with height $h=0,24 \mathrm{~m}$, width $b=0,14 \mathrm{~m}$ and length of $\mathrm{L}=2,4 \mathrm{~m}$. The material has a Young Modulus of $\mathrm{E}=2,5 \times 10^{10} \mathrm{~N} / \mathrm{m}^{2}$ and a material density of $\rho=2,5 \times 10^{3} \mathrm{~kg} / \mathrm{m}^{3}$. The simplicity of this example resembles an experimental prototype in reduced scale that is being tested at the laboratory and that shortly will be used as benchmark for the proposed algorithms.

Figure 3 shows the evolution with generations of the damage extent on elements of the best individual of the population. It can be seen that at $37^{\text {th }}$ generation the damaged site is located and at the $90^{\text {th }}$ generation the damage extent is correctly evaluated.

As indicated by Table 1, it was investigated six more sceneries of damage in the beam. The damaged was allowed to occur on different elements and on more than one site.

The cases indicated in Table 1 were simulated numerically and the first five natural frequencies were used to feed the Genetic Algorithm as a basis for the objective function construction. Figure 4 shows the results for the tested sceneries. Except for case 7, all other sceneries have been corrected located and the damaged extent corrected evaluated. In the 7 th. case the extent of the damage was successfully obtained but the location indicated was one element beside the correct element, as indicated by Fig. 4. 


\section{Conclusions}

This paper has presented a methodology to evaluate damage extent and location by natural frequencies shifts. The formulation uses a genetic real coded algorithm as an optimization tool to match damaged natural frequencies with those obtained by a finite element code through MDLAC index.

The methodology has shown robust, since no approximation (exception for discretization or linear assumptions) is taken for the structural dynamic behavior. This bypass some non linear behavior of the frequencies shifts with increasing damage. In the other hand, the computational cost may be enlarged with problem's complexity.

Damage occurring on one site and moderate extent was successfully found using only first five natural frequencies. Damage occurring on more than one site was accurately evaluated but the location was shifted by one element from the expected ones.

Ever since, some improvements on the optimization tool has been implemented and new structural models has been tested. Some experimental tests are being accomplished and this will be added to the comparisons to validate the model in future works. 